\title{
Predictors of baseline cancer-related cognitive impairment in cancer patients scheduled for a curative treatment
}

\author{
Michelle Lycke ${ }^{1}$ | Lies Pottel ${ }^{1}$ | Hans Pottel ${ }^{2}$ | Lore Ketelaars ${ }^{3}$ | Karin Stellamans ${ }^{4}$ | \\ Koen Van Eygen ${ }^{1,5}$ | Philippe Vergauwe ${ }^{6}$ | Patrick Werbrouck ${ }^{7}$ | Laurence Goethals ${ }^{4}$ | \\ Patricia Schofield ${ }^{8}$ | Tom Boterberg 9 | Philip R. Debruyne T,8* $^{1,{ }^{*}}$
}

${ }^{1}$ Division of Medical Oncology, Cancer Centre, General Hospital Groeninge, Kortrijk, Belgium

${ }^{2}$ Department of Public Health and Primary Care @ Kulak, Catholic University Leuven Kulak, Kortrijk, Belgium

${ }^{3}$ Department of Neuropsychology, General Hospital Groeninge, Kortrijk, Belgium

${ }^{4}$ Division of Radiotherapy, Cancer Centre, General Hospital Groeninge, Kortrijk, Belgium

${ }^{5}$ Division of Haematology, Cancer Centre, General Hospital Groeninge, Kortrijk, Belgium

${ }^{6}$ Department of Gastro-Enterology, General Hospital Groeninge, Kortrijk, Belgium

${ }^{7}$ Department of Urology, General Hospital Groeninge, Kortrijk, Belgium

${ }^{8}$ Faculty of Health, Social Care and Education, Anglia Ruskin University, Chelmsford, UK

${ }^{9}$ Department of Radiation Oncology, Ghent University Hospital, Ghent, Belgium

Correspondence

Prof Philip R. Debruyne, Cancer Centre, General Hospital Groeninge, Pres. Kennedylaan 4, B-8500 Kortrijk, Belgium. Email: philip.debruyne@azgroeninge.be

\begin{abstract}
Introduction Recent research in the field of cancer-related cognitive impairments (CRCI) has shown $\mathrm{CRCl}$ presentation prior to treatment initiation. Some have attributed these problems to worry and fatigue, whereas others have suggested an influence of age, IQ, and other psychosocial and medical factors.

Methods Patients ( $\geq 18$ years) with a histologically confirmed diagnosis of a solid cancer or hematological malignancy, scheduled for a curative treatment, were evaluated with a baseline neuropsychological assessment including Patient-Reported Outcome Measures (PROMs). PROMs entailed distress, anxiety and depression, fatigue, and cognitive complaints. The neuropsychological assessment comprised several cognitive domains such as premorbid IQ, attention, processing speed, flexibility, verbal and visual episodic memory, and verbal fluency.
\end{abstract}

Results Cross-sectional data of 125 patients were collected. Patients had a mean age of 60.9 years (range: $30.0-85.0$ ) and comprised primarily females (65.6\%). Patients presented with cancer of following sites: breast (44.0\%), digestive (28.8\%), urological (11.2\%), gynecologic (8.0\%), hematologic malignancy (4.8\%), and lung (3.2\%). Patients presented with a premorbid IQ of 105.3 (range: $79.0-124.0$ ). In $29.6 \%$ of patients, a CRCI was detected. Binary logistic regression analyses showed that a lower premorbid IQ $(\beta=-.084, P<.01)$ and a higher level of fatigue $(\beta=-.054, P<.05)$ predicted baseline $C R C I$. Premorbid IQ also predicted performance on individual cognitive domains. Some domains were also influenced by age, gender, having a breast cancer diagnosis, and an active treatment for hypertension.

Conclusion Premorbid IQ and fatigue are important predictors of baseline $\mathrm{CRCI}$. Therefore, we advise researchers to implement a short IQ test when conducting clinical trials on $\mathrm{CRCI}$.

\section{KEYWORDS}

baseline, cancer, cancer-related cognitive impairment, chemobrain, cognition, oncology

\section{1 | INTRODUCTION}

Improved cancer treatments have led to increased survival rates and a growing number of cancer survivors presenting with persistent treatment-related side effects. Cognitive malfunctioning is one of the most frequently reported adverse events and poses a big challenge for patients who want to return to their former lives. Patients may suffer from concentration problems, distractibility, forgetfulness, difficulties in remembering names or numbers, and a lack of mental sharpness. ${ }^{1-5}$
Researchers ascribed these problems at first to chemotherapeutic treatments, resulting in a term called "chemobrain." Initial trials focused on breast cancer patients, as they reported symptoms even long after their treatment had ended. ${ }^{2,3}$ Recent research, however, indicates that chemotherapeutic agents may not be the sole cause of cancer-related cognitive impairments ( $\mathrm{CRCl}$ ). Studies have shown that radiotherapy, external to the brain region, and hormonal treatments can also induce $\mathrm{CRCl}^{6-8}$ Further, prospective studies, including neuropsychological assessments before treatment administration but after cancer 
diagnosis, and most often after cancer surgery, have reported high rates of $\mathrm{CRCl}$ prior to adjuvant chemotherapy. They report increasing problems following chemotherapy and a resolution of the findings to baseline levels when performing longer follow-up assessments. ${ }^{1}$

Although the majority of studies now include a baseline assessment that shows that some patients present with a $\mathrm{CRCl}$ before adjuvant treatment initiation, little is known about why these impairments occur. A trial by Schilder et $\mathrm{al}^{8}$ investigated baseline cognition in a group of postmenopausal breast cancer patients and found that an individual cognitive domain can be influenced by age, IQ, and other medical factors. Others have suggested that psychosocial factors such as worry and fatigue may enhance the risk of presenting with a $\mathrm{CRCl}$ at baseline in patients diagnosed with breast or colorectal cancer. $^{9-11}$

Although more research has been conducted into the pathophysiology of baseline $\mathrm{CRCl}$, sufficient evidence is lacking. Further, studies have mainly been focusing on how breast cancer patients experience these problems. It is only until more recently that researchers have broadened their landscape and started to examine $\mathrm{CRCl}$ in other cancer types. Another shortcoming in current literature is that only few researchers implement some form of IQ assessment when investigating $\mathrm{CRCl}$, although it is known that IQ can predict neuropsychological assessment results. ${ }^{12}$

In this paper, we tried closing the gap in some of these shortcomings by performing a cross-sectional analysis in which we aimed at identifying predictors of baseline $\mathrm{CRCl}$ in a group of general cancer patients who were scheduled for a treatment with curative intent.

\section{2 | METHODS}

\section{1 | Participants}

Patients were invited to participate in the CONCEPT-trial (ClinicalTrials.gov Identifier: NCT01846260) between May 2012 and
September 2015. Baseline data were collected as part of an ongoing longitudinal trial in which we aim to examine whether the distress thermometer can predict long-term $\mathrm{CRCl}$ (to be presented in future manuscript). All patients were recruited in the Kortrijk Cancer Centre (Kortrijk, Belgium). Eligible patients were 18 years or older and native Dutch speaking or bilingual. All patients had a histologically confirmed diagnosis of a solid tumor or hematological malignancy, in an early or advanced stage. Patients were scheduled to receive a treatment with curative intent. Patients receiving surgery as a sole treatment were excluded. Other exclusion criteria entailed the following: being diagnosed with primary brain tumors or brain metastases, having a prior history of cancer-with or without chemotherapy or radiotherapy-during the last 5 years, suffering from an organic brain syndrome, showing signs of mental deterioration or being diagnosed with dementia (DSM-IV criteria), having an untreated or unstable major medical condition, being alcohol or drug dependent, presenting with a condition other than cancer in which fatigue is a prominent symptom (such as chronic fatigue syndrome), and having a major psychiatric or neurologic disorder that could potentially invalidate assessment; a prior or current diagnosis of a depressive or anxiety disorder was allowed. All patients gave written informed consent. The trial was approved by the ethics committee of the General Hospital Groeninge, Kortrijk, Belgium.

\section{2 | Measures}

Patients were evaluated by a baseline neuropsychological assessment including Patient-Reported Outcome Measures (PROMs). All assessments were performed by either a neuropsychologist or study trial coordinators trained to perform these measurements. The neuropsychological assessment included standardized neuropsychological tests assessing several cognitive domains (Table 1) as advised by the International Cognition and Cancer Task Force (ICCTF). ${ }^{13}$

The Dutch Adult Reading Test (DART) is the Dutch version of the National Adult Reading Test. It consists of a list of 50 words with an

TABLE 1 Neuropsychological assessment

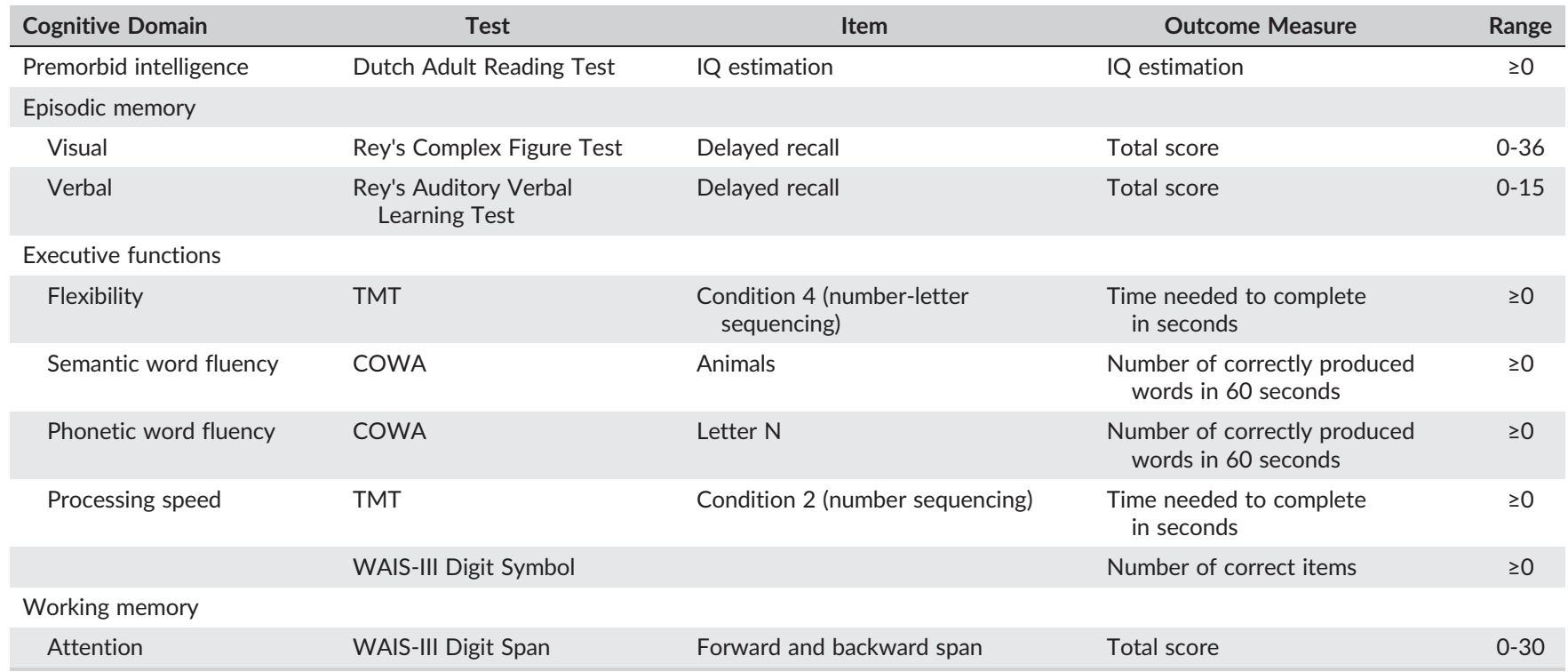

Abbreviations: COWA, Controlled Oral Word Association Test; TMT, Trail Making Test; WAIS, Wechsler Adult Intelligence Scale. 
irregular pronunciation that have to be read out loud. The DART estimates premorbid intelligence and is relatively insensitive to brain dysfunctions and mild dementia. ${ }^{14,15}$

The Rey-Osterrieth Complex Figure Test (CFT) assesses both visuoconstruction and visual memory. ${ }^{16,17}$ It consists of 3 conditions: a copy task, an immediate task, and a delayed recall task. The CFT has been a useful tool for measuring visual episodic memory that is mediated by the prefrontal lobe. ${ }^{18}$

The Rey Auditory Verbal Learning Test (RAVLT) measures verbal learning ability and verbal memory. Patients are asked to repeat and remember a list of 15 words. It entails both immediate and delayed recall tasks. ${ }^{19}$

The Trail Making Test (TMT) provides information on a patient's visual scanning and searching abilities, processing speed, mental flexibility, and executive function. ${ }^{20}$ Delis-Kaplan Executive Function System TMT consists of 5 conditions instead of 2 on the original test. Patients are asked to draw lines sequentially connecting encircled numbers or letters distributed on a sheet of paper. The most important conditions concerning executive functioning comprises number and number-letter sequencing tasks. ${ }^{21}$

The Controlled Oral Word Association (COWA) test is one of the most commonly used measures of verbal fluency. This rapid and organized word retrieval task is a sensitive indicator of brain dysfunctions. Verbal fluency tests typically employ a word-list generation procedure and are divided into 2 forms. Semantic fluency tasks require the patient to generate a list of words according to a certain category. Phonemic fluency tasks require that words are generated according to a letter of the alphabet. ${ }^{22-24}$

The Digit Span subtest of Wechsler Adult Intelligence Scale-III (WAIS-III) measures attention, concentration, and working memory and entails forward and backward repeating tasks. The score is the total number of correctly repeated sequences before 2 failed attempts in each condition. ${ }^{25,26}$ The WAIS-III Digit Symbol measures cognitive and perceptual-motor processing speed. The patient is given a code that pairs symbols with digits. The patient is asked to match as many series of digits as possible to their corresponding symbols as possible in a fixed time span of 120 seconds. ${ }^{26,27}$

PROMs entailed an assessment of distress (distress thermometer including 38-item Problem list ${ }^{28}$ ), anxiety and depression (Hospital Anxiety and Depression Scale ${ }^{29}$ ), fatigue (FACIT-Fatigue ${ }^{30}$ ), cognitive complaints (Cognitive Failure Questionnaire ${ }^{31}$ ), and quality of life (EORTC QLQ-C30 32 ).

\section{3 | Statistical considerations}

Statistical analyses were conducted by use of SPSS software (version 23; IBM SPSS Statistics, IBM, Chicago, Illinois). Descriptive statistics were performed to present patient and tumor characteristics and neuropsychological assessment results. Overall cognitive impairment was calculated by the definition of the ICCTF. Patients were marked as having a $\mathrm{CRCl}$ if they presented with either 2 or more test scores at or below -1.5 standard deviations (SDs) from the normative mean or if they presented with 1 test score at or below -2.0 SDs. ${ }^{13}$ Published normative data, adjusted for gender, age, and/or education, were used to convert raw test scores into standardized $z$-scores (mean $=0 ; S D=1$ ). Curves based on the binomial probability distribution were used to determine that in our test battery, including 8 independent test, approximately $17 \%$ of patients would perform 2 SDs below the normative mean on a single test. ${ }^{33} \mathrm{~A}$ binomial test was performed to examine whether our data differed from the binomial probability distribution. Data from questionnaires were converted according to standard scoring rules, if applicable.

Independent Student $t$ and $\chi^{2}$ tests were performed to examine patient and clinical characteristics between impaired and nonimpaired participants. Binary logistic regression analysis was used to examine potential predictors of overall $\mathrm{CRCl}$. Multiple regression analysis was used to examine predictors of individual cognitive domains. Models were selected through forward and backward analyses. Both binary and linear regression analyses included 14 covariates: age, gender, premorbid IQ, distress, fatigue, cognitive complaints, days since diagnosis, days since surgery, active treatment for diabetes mellitus, active treatment for hypertension, active treatment with anxiolytics/antidepressants/antihypnotics, having a prior or current diagnosis of depression or anxiety, stage (early vs late stage), and diagnosis (breast cancer or not). Variables were included in the model if they were significant at the $P<.05$ level.

\section{3 | RESULTS}

\section{1 | Patient characteristics}

In total, 125 patients were included in the trial. Patients had a mean age of 60.9 years (range: $30.0-85.0$ ). The study population comprised primarily female individuals (65.6\%). The majority of patients finished high school or higher (71.2\%). Patients presented with cancer of following sites: breast (44.0\%), digestive (28.8\%), urological (11.2\%), gynecologic (8.0\%), hematologic malignancy $(4.8 \%)$, or lung $(3.2 \%)$. Most patients were diagnosed in an early stage (62.4\%). Eighty-six patients underwent surgery prior to the baseline assessment. On average, there were 38.1 days (range: 13-106) between the day of surgery and the day of the assessment. Five patients were included with a prior history of diagnosed depression or anxiety disorder. No patient was included with a current diagnosis of any of these conditions. Of all patients, $22.4 \%$ were prescribed antidepressants, antihypnotics, and/or anxiolytics. Only few patients received an active treatment for diabetes mellitus (6.4\%), whereas almost half of patients were on antihypertensive drugs (42.4\%) (Table 2 ).

\section{2 | Neuropsychological outcomes}

One patient was excluded from the analyses as not all neuropsychological tests were completed. Table 3 shows mean raw scores, $z$-scores, and SDs for each cognitive test. Patients had a mean premorbid IQ of 105.5 (range: 79.0-124.0). Based on the definition of the ICCTF, $29.6 \%$ of patients presented with an overall $\mathrm{CRCl}$. Thirty patients scored below 2 SDs from the normative mean on a single test $(24.2 \%$, binomial test $P<.001$ ). Independent Student $t$ tests did not detect differences between impaired and nonimpaired patients for age, education age, distress, anxiety, depression, fatigue, subjective cognitive complaints, and days between surgery and baseline assessment, nor did the $\chi^{2}$ test 
TABLE 2 Demographic and clinical data $(n=125)$

\begin{tabular}{|c|c|c|}
\hline & n (\%) & Mean (Range) \\
\hline \multicolumn{3}{|l|}{ Demographics } \\
\hline Age & & $60.9(30.0-85.0)$ \\
\hline \multicolumn{3}{|l|}{ Gender } \\
\hline Female & $82(65.6)$ & \\
\hline Male & $43(34.4)$ & \\
\hline \multicolumn{3}{|l|}{ Highest education } \\
\hline Primary education & $0(0)$ & \\
\hline Lower secondary education & $36(28.8)$ & \\
\hline Higher secondary education & $49(39.2)$ & \\
\hline Higher education & $35(28.0)$ & \\
\hline Other & $5(4.0)$ & \\
\hline \multicolumn{3}{|l|}{ Clinical data } \\
\hline \multicolumn{3}{|l|}{ Diagnosis } \\
\hline Breast cancer & $55(44.0)$ & \\
\hline Digestive cancer & $36(28.8)$ & \\
\hline Urological cancer & $14(11.2)$ & \\
\hline Gynecologic cancer & $10(8.0)$ & \\
\hline Hematologic malignancy & $6(4.8)$ & \\
\hline Lung cancer & $4(3.2)$ & \\
\hline \multicolumn{3}{|l|}{ Stage } \\
\hline Early (I-II) & $78(62.4)$ & \\
\hline Advanced (III-IV) & 47 (37.6) & \\
\hline \multicolumn{3}{|l|}{ Surgery } \\
\hline $\begin{array}{l}\text { Number of patients who received } \\
\text { surgery before baseline assessment }\end{array}$ & $86(68.8)$ & \\
\hline $\begin{array}{l}\text { Days between surgery and } \\
\text { baseline assessment }\end{array}$ & & $38.1(13-106)$ \\
\hline \multicolumn{3}{|l|}{ Medication } \\
\hline Active treatment diabetes mellitus & $8(6.4)$ & \\
\hline Active treatment hypertension & $53(42.4)$ & \\
\hline $\begin{array}{l}\text { Active treatment with anxiolytics/ } \\
\text { antidepressants/antihypnotics }\end{array}$ & $28(22.4)$ & \\
\hline
\end{tabular}

TABLE 3 Mean raw and $z$-scores and SDs per cognitive test $(n=124)$

\begin{tabular}{lcc} 
& Raw score & $z$-score \\
\cline { 2 - 3 } Cognitive Test & Mean (SD) & Mean (SD) \\
\hline DART & $105.3(9.1)$ & NA \\
\hline CFT delayed recall & $19.6(5.1)$ & $0.07(0.76)$ \\
\hline RAVLT delayed recall & $10.3(3.8)$ & $-0.26(1.48)$ \\
\hline $\begin{array}{l}\text { TMT condition 4: number-letter } \\
\quad \text { sequencing }\end{array}$ & $104.7(53.9)$ & $0.06(1.10)$ \\
\hline COWA semantic word fluency & $21.7(7.0)$ & $0.01(1.09)$ \\
COWA phonetic word fluency & $10.0(5.0)$ & $0.00(1.22)$ \\
\hline TMT condition 2: number sequencing & $43.8(22.1)$ & $0.20(1.09)$ \\
WAIS-III Digit Symbol & $63.6(20.4)$ & $0.28(1.21)$ \\
\hline WAIS-III Digit Span & $14.3(3.5)$ & $0.10(1.04)$ \\
\hline
\end{tabular}

Abbreviations: CFT, Complex Figure Test; COWA, Controlled Oral Word Association; DART, Dutch Adult Reading Test; NA, not applicable; RAVLT, Rey's Auditory Verbal Learning Test; SD, standard deviation; TMT, Trail Making Test; WAIS, Wechsler Adult Intelligence Scale.

show any differences between both groups for gender, active treatment with anxiolytics/antidepressants/antihypnotics, having a prior or current diagnosis of depression or anxiety, stage (early vs late stage), or cancer type (breast cancer vs other cancer type) (data not shown). A significant difference was found for premorbid IQ $(P<.01)$. Nonimpaired patients presented with a mean premorbid IQ of 107.0 (range, 79.0-124.0), whereas the mean premorbid IQ of impaired patients was calculated as 101.5 (range: 82.0-116.0).

All regression analyses started with a list of 14 covariates as mentioned previously. Results of the binary logistic regression analyses indicated that overall $\mathrm{CRCl}$, according to the definition of the ICCTF, was predicted by a lower premorbid IQ $(\beta=-.084, P<.01)$ and lower score on the FACIT-Fatigue scale representing a higher level of fatigue $(\beta=-.054, P<.05)$. Individual cognitive domains were evaluated through multiple regression analysis (Table 4). Results revealed that all cognitive domains can be predicted by premorbid IQ, stating that a higher IQ results in a better test score. Premorbid IQ alone predicted up to $27.1 \%$ of the explained variance ( $R^{2}$ adjusted) in a single test domain. Visual and verbal episodic memory, information processing speed, semantic word fluency, and flexibility were also influenced by age, favoring younger patients. Including age in the model resulted in an up to $31.7 \%$ increase of the explained variance. Verbal episodic memory was further predicted by gender resulting in a total explained variance of 33.2\%. Test scores on the WAIS-III Digit Span were, next to premorbid IQ, predicted by an active treatment for hypertension, adding $8.0 \%$ to the explained variance of the model. Interestingly, processing speed, as measured by the WAIS-III Digit Symbol, was in part predicted by having a breast cancer diagnosis or not.

\section{4 | DISCUSSION}

This paper aimed at identifying risk factors for baseline $\mathrm{CRCl}$ in a group of general cancer patients scheduled for a curative treatment. Our data highlight the importance of conducting an IQ test when conducting neuropsychological assessments in cancer patients. Results indicated that $\mathrm{CRCl}$, which is defined as presenting with 2 or more test scores at or below -1.5 SDs from the normative mean or presenting with 1 test score at or below -2.0 SDs, was predicted by premorbid IQ and fatigue. Further, individual neuropsychological test scores were all influenced by premorbid IQ. Some cognitive domains were also predicted by gender, age, having a breast cancer diagnosis or not, and/or an active treatment for hypertension.

Our results indicate that IQ predicts baseline $\mathrm{CRCI}$. To our knowledge, we are the first to report this finding in case of overall $\mathrm{CRCl}$. Our data also indicate that IQ influences individual cognitive domains. These results are in line with previous literature as the IQ of a patient has been reported as a strong predictor of neuropsychological test scores in both cancer and noncancer participants. Diaz-Asper et $\mathrm{al}^{34}$ evaluated the influence of IQ on several individual cognitive tests in 221 normal adults and stated that IQ predicts concurrent neuropsychological performance across the entire spectrum of intelligence. In a group of breast cancer patients exposed to chemotherapy, Ahles et al ${ }^{35}$ reported that pretreatment cognitive reserve, assessed by the Wide Range Achievement Test-3 (WRAT-3), was related with post-treatment cognitive decline. Further, the data of Schilder et al are in accordance with our findings. They reported IQ to be a predictor of individual cognitive domains in a group of 
TABLE 4 Multiple regression analysis

\begin{tabular}{|c|c|c|c|c|}
\hline Models & $R^{2}$ Adj. & $\begin{array}{l}\text { Dependent Variables } \\
\text { in Final Models }\end{array}$ & $\beta$ & $P$ Value \\
\hline \multicolumn{5}{|l|}{ CFT delayed recall } \\
\hline IQ & 0.140 & IQ & 0.328 & $<0.001$ \\
\hline IQ + age & 0.204 & Age & -0.270 & $<0.01$ \\
\hline \multicolumn{5}{|l|}{ RAVLT delayed recall } \\
\hline IQ & 0.223 & IQ & 0.421 & $<0.001$ \\
\hline IQ + gender + age & 0.332 & Gender & 0.204 & $<0.01$ \\
\hline \multicolumn{5}{|l|}{ TMT number-letter sequencing } \\
\hline IQ & 0.186 & IQ & -0.325 & $<0.001$ \\
\hline IQ + age & 0.467 & Age & 0.544 & $<0.001$ \\
\hline \multicolumn{5}{|l|}{ COWA semantic word fluency } \\
\hline $\mathrm{IQ}$ & 0.271 & IQ & 0.526 & $<0.001$ \\
\hline \multicolumn{5}{|l|}{ TMT: number sequencing } \\
\hline IQ & 0.105 & IQ & -0.224 & $<0.01$ \\
\hline IQ + age & 0.372 & Age & 0.531 & $<0.001$ \\
\hline \multicolumn{5}{|l|}{ WAIS-III Digit Symbol } \\
\hline IQ & 0.170 & IQ & 0.272 & $<0.001$ \\
\hline IQ + age & 0.487 & Age & -0.566 & $<0.001$ \\
\hline $\mathrm{IQ}+$ age + having breast cancer or not & 0.508 & $\begin{array}{l}\text { Having breast } \\
\text { cancer or not }\end{array}$ & 0.161 & $<0.05$ \\
\hline \multicolumn{5}{|l|}{ WAIS-III Digit Span } \\
\hline
\end{tabular}

Abbreviations: CFT, Complex Figure Test; COWA, Controlled Oral Word Association; RAVLT, Rey's Auditory Verbal Learning Test; TMT, Trail Making Test; WAIS, Wechsler Adult Intelligence Scale.

Covariates: age, gender, premorbid IQ, distress, fatigue, cognitive complaints, days since diagnosis, days since surgery, active treatment for diabetes mellitus, active treatment for hypertension, active treatment with anxiolytics/antidepressants/antihypnotics, having a prior or current diagnosis of depression or anxiety, stage (early vs late stage), and diagnosis (breast cancer or not).

postmenopausal breast cancer patients before the administration of adjuvant systemic treatment. ${ }^{8}$ Lange et al, $^{36}$ however, examined baseline cognition in older cancer patients and could not detect any correlations between $\mathrm{CRCl}$ and clinical characteristics. When comparing our data with the binomial probability distribution, we detected a statistical significant result $(P<.001)$ stating that the number of impaired $\mathrm{CRCI}$ can only in part be explained by normal variance.

In our study, overall $\mathrm{CRCl}$ was also predicted by fatigue. Although it has been noted that fatigue influences subjective cognitive complaints in cancer patients, most studies have failed to find an association between objective $\mathrm{CRCl}$ and fatigue. ${ }^{37-39}$ Booth-Jones et al ${ }^{40}$ examined the cognitive function of patients who underwent a bone marrow transplantation and reported that both objective and subjective cognitive impairments are influenced by the level of fatigue. Further, recent research by Menning et $\mathrm{al}^{11}$ found that symptoms of fatigue were related to observed impairments in breast cancer patients when compared with healthy controls, prior to adjuvant treatment.

Our data suggest that age could predict processing speed, executive function, verbal episodic memory, and semantic word fluency. This finding is in accordance with the results of Lange et $\mathrm{al}^{36}$ who examined baseline cognition in older breast cancer patients. They reported that more than $40 \%$ presented with a $\mathrm{CRCl}$ at baseline and that respectively $15 \%, 16 \%$, and $21 \%$ of patients presented with an impairment in the domain of processing speed, executive function, and verbal episodic memory. ${ }^{36}$ Further, age-related decline on cognitive functioning has also been noted in noncancer participants. ${ }^{41}$ For example, Kramer et al ${ }^{42}$ stated that older healthy participants can present with poorer verbal memory results when compared with their younger counterparts.

Verbal episodic memory, as measured by the RAVLT delayed recall, was also predicted by gender. These findings are in line with those of Kramer et $\mathrm{al}^{42}$ who found comparable results in a group of healthy individuals. In their study, they have noted that men perform worse on a delayed recall test. ${ }^{42}$

Our data further indicate that an active treatment for hypertension predicts in part the outcome on the WAIS-III Digit Span, which measures attention. It is known that hypertension influences cognitive performance. Knecht et $\mathrm{al}^{43}$ reported that hypertension may account for one-tenth of the cognitive impairments in nondemented community-dwelling participants. Schilder et $\mathrm{al}^{8}$ confirm this finding in a group 
of postmenopausal breast cancer patients. This finding is a reminder that cancer occurs within the context of multiple comorbidities that could each have its own influence on the patient's cognitive abilities and that it is important to take these into account when conducting clinical trials on $\mathrm{CRCl}$.

A breast cancer diagnosis seems to affect performance on the WAIS-III Digit Symbol. Although we could not find evidence to support this finding at baseline, Schagen et $\mathrm{al}^{44}$ reported that breast cancer patients who were treated with chemotherapy (cyclophosphamide, methotrexate, and 5-fluorouracil, a somewhat outdated treatment scheme nowadays) performed worse on the WAIS Digit Symbol than breast cancer patients who did not receive chemotherapy. As previously mentioned, research on $\mathrm{CRCl}$ mainly focuses on breast cancer patients. A possible explanation for this may be that breast cancer patients are more emotionally open and express side effects quicker than others. In our trial, comprising $44.0 \%$ breast cancer patients, we found that-although not statistical significant-breast cancer patients experienced more subjective cognitive complaints than other cancer patients. On the contrary, a fewer percentage of breast cancer patients than others were found to have $\mathrm{CRCl}$ (not significant data not shown).

The strengths of this study include several aspects. First, although it is also listed as a limitation, we did include several cancer types. It is known that most research on $\mathrm{CRCl}$ is performed in breast cancer patients and that this is a shortcoming in current literature. Although more researcher have gained interest in other cancer types, highlighting that not solely breast cancer patients experience $\mathrm{CRCl}$ remains important. Further, as a result of including a high number of breast cancer patients, we were able to use this as a covariate in our analysis, making it possible to see if breast or rather other cancer patients are more prone to certain cognitive impairments. Second, we used the DART to examine IQ, which is a quick and easy assessment tool. Other trials, investigating mainly postadjuvant treatment $\mathrm{CRCl}$, used tests such as the WRAT. ${ }^{35,45}$ Although clinically useful to screen for premorbid intelligence, the WRAT can take up to 45 minutes to administer depending on the age the patient, therefore making it less useful to add to an already exhaustive list of neuropsychological tests. Third, this trial includes a wide range of cognitive domains and implements a number of tests that are advised by the ICCTF. ${ }^{13}$ Further, we also chose to use their definition of $\mathrm{CRCl}$ to facilitate comparing trial results with others. Last, our study tried to confirm findings of the few researchers who have reported predictors of baseline $\mathrm{CRCl}$ in cancer patients.

The results of our analysis need to be interpreted with caution. First, we did not include a healthy control group. Nevertheless, we compared our findings with the binomial probability distribution. We estimated that approximately $17 \%$ of patients would score at least 2 SDs below the normative mean on a single test score when using a neuropsychological assessment including 8 independent tests. Results found a statistical significant difference indicating that our selected population differs from healthy participants, thus only in part explaining the influence of $\mathrm{IQ}$, which is a known confounder of neuropsychological tests. ${ }^{12}$ Second, we have included patients of all cancer types and did not find a normal distribution across the cancer types. Although we believe that it is necessary to perform these studies in patients diagnosed with all cancer types, it may mask certain differences. Nonetheless, statistical analysis revealed that the cancer type did not influence overall impairment. Having a breast cancer diagnosis did influence the outcome on the WAIS Digit Symbol. Further research is warranted to compare breast and other cancer patients. Third, some neuropsychological tests, such as the RAVLT and CFT, did not provide optimal z-scores for older patients. $z$-scores can only be calculated in 3 age categories ( $>30,30-50$, and $<50$ years), which may result in more impairments in older patients, because of this shortcoming in the normative data. On the other hand, when conducting the regression analyses, age was included as a covariate. Further, the linear regression analyses used raw test score instead of $z$-scores. Raw scores were selected to be able to compare our results with findings of other researchers. Therefore, the age and IQ effect may be more present in these results. Nonetheless, when using the standardized z-scores, IQ effects remain present in all domains. The influence of age remains present in the RAVLT and both conditions of the TMT (data not shown).

To the best of our knowledge, this paper is the first to report baseline cognition of a heterogeneous group of cancer patients scheduled to receive a curative treatment. Although future research is needed to confirm our findings regarding medical and psychosocial factors such as fatigue in particular, we advise other researchers to include a short IQ evaluation such as the DART, which is quick and easy to administer, when conducting neuropsychological assessments in clinical trials investigating $\mathrm{CRCl}$.

\section{ACKNOWLEDGMENTS}

Our work was supported by the Cancer Plan Action 21/22C of the Belgian Federal Government stimulating innovative approaches in psychosocial care (KPC_2122C_044).

\section{CONFLICT OF INTEREST}

The authors have no conflict of interest to declare.

\section{REFERENCES}

1. Burstein HJ. Cognitive side-effects of adjuvant treatments. Breast. 2007;16(Suppl 2):S166-S168. doi: 10.1016/j.breast.2007.07.027

2. Koppelmans V, Breteler MM, Boogerd W, Seynaeve C, Gundy C, Schagen SB. Neuropsychological performance in survivors of breast cancer more than 20 years after adjuvant chemotherapy. J Clin Oncol. 2012;30(10):1080-1086. doi: 10.1200/JCO.2011.37.0189

3. Jim HS, Phillips KM, Chait S, et al. Meta-analysis of cognitive functioning in breast cancer survivors previously treated with standard-dose chemotherapy. J Clin Oncol. 2012;30(29):3578-3587. doi: 10.1200/ JCO.2011.39.5640

4. Ketelaars L, Pottel L, Lycke M, et al. Use of the Freund Clock Drawing Test within the Mini-Cog as a screening tool for cognitive impairment in elderly patients with or without cancer. J Geriatr Oncol. 2013;4 (2):174-182. doi: 10.1016/j.jgo.2012.10.175

5. Lycke M, Ketelaars L, Boterberg T, et al. Validation of the Freund Clock Drawing Test as a screening tool to detect cognitive dysfunctions in elderly cancer patients undergoing comprehensive geriatric assessment. Psycho-Oncology. 2014;23:114-114.

6. Fuller CD, Schillerstrom JE, Jones WE 3rd, Boersma M, Royall DR, Fuss M. Prospective evaluation of pretreatment executive cognitive 
impairment and depression in patients referred for radiotherapy. Int J Radiat Oncol Biol Phys. 2008;72(2):529-533. doi: 10.1016/j. ijrobp.2007.12.040

7. Gonzalez BD, Jim HS, Booth-Jones M, et al. Course and predictors of cognitive function in patients with prostate cancer receiving androgen-deprivation therapy: a controlled comparison. J Clin Oncol. 2015;33(18):2021-2027. doi: 10.1200/JCO.2014.60.1963

8. Schilder CM, Seynaeve C, Beex LV, et al. Effects of tamoxifen and exemestane on cognitive functioning of postmenopausal patients with breast cancer: results from the neuropsychological side study of the tamoxifen and exemestane adjuvant multinational trial. J Clin Oncol. 2010;28(8):1294-1300. doi: 10.1200/JCO.2008.21.3553

9. Berman MG, Askren MK, Jung M, et al. Pretreatment worry and neurocognitive responses in women with breast cancer. Health Psychol. 2014;33(3):222-231. doi: 10.1037/a0033425

10. Visovatti MA, Reuter-Lorenz PA, Chang AE, Northouse L, Cimprich B. Assessment of cognitive impairment and complaints in individuals with colorectal cancer. Oncol Nurs Forum. 2016;43(2):169-178. doi: 10.1188/16.ONF.43-02AP

11. Menning S, de Ruiter MB, Veltman DJ, et al. Multimodal MRI and cognitive function in patients with breast cancer prior to adjuvant treatment-the role of fatigue. Neuroimage Clin. 2015;7:547-554. doi: 10.1016/j.nicl.2015.02.005

12. Diaz-Asper CM, Schretlen DJ, Pearlson GD. How well does IQ predict neuropsychological test performance in normal adults? J Int Neuropsychol Soc. 2004;10(1):82-90.

13. Wefel JS, Vardy J, Ahles T, Schagen SB. International Cognition and Cancer Task Force recommendations to harmonise studies of cognitive function in patients with cancer. Lancet Oncol. 2011;12(7):703-708. doi: 10.1016/S1470-2045(10)70294-1

14. Schmand B, Smit JH, Geerlings MI, Lindeboom J. The effects of intelligence and education on the development of dementia. A test of the brain reserve hypothesis. Psychol Med. 1997;27(6):1337-1344.

15. Bright P, Jaldow E, Kopelman MD. The National Adult Reading Test as a measure of premorbid intelligence: a comparison with estimates derived from demographic variables. J Int Neuropsychol Soc. 2002;8 (6):847-854.

16. Osterrieth PA. Test of copying a complex figure; contribution to the study of perception and memory. Arch Psychol. 1944;30:206-356.

17. Rey A. The psychological examination in cases of traumatic encepholopathy. Arch Psychol. 1941;28:215-285.

18. Shin MS, Park SY, Park SR, Seol SH, Kwon JS. Clinical and empirical applications of the Rey-Osterrieth Complex Figure Test. Nat Protoc. 2006;1(2):892-899. doi: 10.1038/nprot.2006.115

19. Schmidt M. Rey Auditory and Verbal Learning Test: A Handbook. Los Angeles, CA, USA: Western Psychological Services;1996.

20. Tombaugh TN. Trail Making Test A and B: normative data stratified by age and education. Arch Clin Neuropsychol. 2004;19(2):203-214. doi: 10.1016/S0887-6177(03)00039-8

21. Delis DC, Buysch H, Op den Noens IL, Berckelaer-Onnes IA, Kaplan E, Kramer JH. D-KEFS: Delis-Kaplan Executive Function System: Trail Making Test. Amsterdam: Harcourt Test Publishers;2007.

22. Ruff RM, Light RH, Parker SB, Levin HS. Benton Controlled Oral Word Association Test: reliability and updated norms. Arch Clin Neuropsychol. 1996;11(4):329-338.

23. Troyer AK, Moscovitch M, Winocur G. Clustering and switching as two components of verbal fluency: evidence from younger and older healthy adults. Neuropsychology. 1997;11(1):138-146.

24. Troyer AK, Moscovitch M, Winocur G, Leach L, Freedman M. Clustering and switching on verbal fluency tests in Alzheimer's and Parkinson's disease. J Int Neuropsychol Soc. 1998;4(2):137-143.

25. Wilde N, Strauss E. Functional equivalence of WAIS-III/WMS-III digit and spatial span under forward and backward recall conditions. Clin Neuropsychol. 2002;16(3):322-330. doi: 10.1076/ clin.16.3.322.13858

26. Wechsler D. Wechsler Adult Intelligence Scale-Third Edition. Assessment and Scoring Manual. Amsterdam: Harcourt Test Publishers;1997 , 1998, 2000, 2002, 2004.

27. Davis AS, Pierson EE. The relationship between the WAIS-III digit symbol Coding and executive functioning. Appl Neuropsychol Adult. 2012;19(3):192-197. doi: 10.1080/09084282.2011.643958

28. Donovan KA, Grassi L, McGinty HL, Jacobsen PB. Validation of the distress thermometer worldwide: state of the science. Psycho-Oncology. 2014;23(3):241-250. doi: 10.1002/pon.3430

29. Zigmond AS, Snaith RP. The hospital anxiety and depression scale. Acta Psychiatr Scand. 1983;67(6):361-370.

30. Cella D, Yount S, Sorensen M, Chartash E, Sengupta N, Grober J. Validation of the Functional Assessment of Chronic Illness Therapy Fatigue Scale relative to other instrumentation in patients with rheumatoid arthritis. J Rheumatol. 2005;32(5):811-819.

31. Broadbent DE, Cooper PF, FitzGerald P, Parkes KR. The Cognitive Failures Questionnaire (CFQ) and its correlates. Br J Clin Psychol. 1982;21 (Pt 1):1-16.

32. Groenvold M, Klee MC, Sprangers MA, Aaronson NK. Validation of the EORTC QLQ-C30 quality of life questionnaire through combined qualitative and quantitative assessment of patient-observer agreement. J Clin Epidemiol. 1997;50(4):441-450.

33. Ingraham LJ, Aiken CB. An empirical approach to determining criteria for abnormality in test batteries with multiple measures. Neuropsychology. 1996;10(1):120-124. doi: 10.1037/0894-4105.10.1.120

34. Diaz-Asper CM, Schretlen DJ, Pearlson GD. How well does IQ predict neuropsychological test performance in normal adults? J Int Neuropsychol Soc. 2004;10(1):82-90. doi: 10.1017/ S1355617704101100

35. Ahles TA, Saykin AJ, McDonald BC, et al. Longitudinal assessment of cognitive changes associated with adjuvant treatment for breast cancer: impact of age and cognitive reserve. J Clin Oncol. 2010;28 (29):4434-4440. doi: 10.1200/JCO.2009.27.0827

36. Lange M, Giffard B, Noal S, et al. Baseline cognitive functions among elderly patients with localised breast cancer. Eur J Cancer. 2014;50 (13):2181-2189. doi: 10.1016/j.ejca.2014.05.026

37. Mehnert A, Scherwath A, Schirmer L, et al. The association between neuropsychological impairment, self-perceived cognitive deficits, fatigue and health related quality of life in breast cancer survivors following standard adjuvant versus high-dose chemotherapy. Patient Educ Couns. 2007;66(1):108-118. doi: 10.1016/j. pec.2006.11.005

38. Vardy J, Dhillon HM, Pond GR, et al. Cognitive function and fatigue after diagnosis of colorectal cancer. Ann Oncol. 2014;25(12):2404U221. doi: 10.1093/annonc/mdu448

39. Skaali T, Fossa SD, Andersson S, et al. Self-reported cognitive problems in testicular cancer patients: relation to neuropsychological performance, fatigue, and psychological distress. J Psychosom Res. 2011;70 (5):403-410. doi: 10.1016/j.jpsychores.2010.12.004

40. Booth-Jones M, Jacobsen PB, Ransom S, Soety E. Characteristics and correlates of cognitive functioning following bone marrow transplantation. Bone Marrow Transplant. 2005;36(8):695-702. doi: 10.1038/sj. bmt.1705108

41. Kramer J. Pathways to age-related cognitive decline. Ann Neurol. 2013;73(5):563-564. doi: 10.1002/ana.23889

42. Kramer JH, Yaffe K, Lengenfelder J, Delis DC. Age and gender interactions on verbal memory performance. J Int Neuropsychol Soc. 2003;9 (1):97-102. doi: 10.1017/S1355617703910113

43. Knecht S, Wersching H, Lohmann H, Berger K, Ringelstein EB. How much does hypertension affect cognition?: explained variance in cross-sectional analysis of non-demented community-dwelling 
individuals in the SEARCH study. J Neurol Sci. 2009;283(1-2):149-152. doi: 10.1016/j.jns.2009.02.362

44. Schagen SB, van Dam FS, Muller MJ, Boogerd W, Lindeboom J, Bruning PF. Cognitive deficits after postoperative adjuvant chemotherapy for breast carcinoma. Cancer. 1999;85(3):640-650.

45. Ahles TA, Saykin AJ, McDonald BC, et al. Cognitive function in breast cancer patients prior to adjuvant treatment. Breast Cancer Res Treat. 2008;110(1):143-152. doi: 10.1007/s10549-007-9686-5
How to cite this article: Lycke, M., Pottel, L., Pottel, H., Ketelaars, L., Stellamans, K., Van Eygen, K., Vergauwe, P., Werbrouck, P., Goethals, L., Schofield, P., Boterberg, T., and Debruyne, P. R. (2016), Predictors of baseline cancer-related cognitive impairment in cancer patients scheduled for a curative treatment, Psycho-Oncology, doi: 10.1002/pon.4200 